\title{
CELLULAR LOCALISATION OF THE PROTEINS OF REGION 3 OF FELINE ENTERIC CORONAVIRUS
}

\author{
István MÉSZÁRos ${ }^{1}$, Ferenc OLASZ $^{1}$, Enikő KÁDÁR-HÜRKECZ ${ }^{1,2}$, Ádám BÁLINT ${ }^{3}$, \\ Ákos HORNYÁK ${ }^{3}$, Sándor BELÁK ${ }^{4}$ and Zoltán ZÁDORI ${ }^{*}$ \\ ${ }^{1}$ Institute for Veterinary Medical Research, Centre for Agricultural Research, Hungarian \\ Academy of Sciences, Hungária krt. 21, H-1143 Budapest, Hungary; ${ }^{2}$ National Institute \\ of Environmental Health, Budapest, Hungary; ${ }^{3}$ National Food Chain Safety Office \\ Veterinary Diagnostic Directorate, Budapest, Hungary; ${ }^{4}$ Department of Biomedical \\ Sciences and Veterinary Public Health (BVF), Swedish University of Agricultural \\ Sciences (SLU), Uppsala, Sweden
}

(Received 2 March 2018; accepted 25 July 2018)

Feline enteric coronaviruses have three open reading frames (ORFs) in region 3 ( $3 \mathrm{a}, 3 \mathrm{~b}$, and $3 \mathrm{c}$ ). All three ORFs were expressed with C-terminal eGFP and $3 x$ FLAG tags in different cell lines and their localisation was determined. ORF 3a is predicted to contain DNA-binding and transcription activator domains, and it is localised in the nucleus and in the cytoplasm. ORF $3 \mathrm{~b}$ is also predicted to contain DNA-binding and activator domains, and was found to localise in the mitochondrion. Besides that, in some of the non-infected and FIPV-infected cells nucleolar, perinuclear or nuclear membrane accumulation of the eGFP-tagged $3 \mathrm{~b}$ was observed. The exact compartmental localisation of ORF $3 \mathrm{c}$ is yet to be determined. However, based on our co-localisation studies $3 \mathrm{c}$ does not seem to be localised in the ER-Golgi network, ERGIC or peroxisomes. The expression of 3c-eGFP is clearly cell type dependent, it is more stable in MARC 145 cells than in Fcwf-4 or $\mathrm{CrFK}$ cells, which might reflect in vivo stability differences of $3 \mathrm{c}$ in natural target cells (enterocytes vs. monocytes/macrophages).

Key words: FECV, FIPV, coronavirus, ORF, mitochondrial localisation, nucleolar localisation

Feline enteric coronavirus (FECV) infection is very common in domestic cats all around the world. Approximately 20 to $60 \%$ of cats show seropositivity to the virus. From the two serotypes of FECV, serotype I is more prevalent in the field, causing $80-95 \%$ of the infections. Serotype II FECVs are recombinant viruses in which the serotype I S gene and the surrounding regions are replaced by the equivalent canine coronavirus (CCoV) sequences (Herrewegh et al., 1998).

FECV replication is mostly confined to the intestinal tract, and the infection is usually asymptomatic or may result in mild, self-limiting gastrointestinal disease. As estimated, in around 5\% of FECV-infected animals, a progressive

*Corresponding author; zadori.zoltan@agrar.mta.hu; Phone: 0036 (1) 467-4069 
debilitating disease, feline infectious peritonitis (FIP) develops. FIP is characterised by very high mortality and it is currently the leading infectious cause of death among young domestic cats. It is generally accepted that the basic reason for the development of FIP syndromes is the enhanced replication rate of FIP virus (FIPV) in monocytes and macrophages. In the case of FECV/FIPV, the shifting of the focus of viral replication from the intestinal tract toward macrophages and monocytes usually goes hand in hand with deletions in the genomic regions 3 (Bálint et al., 2014; Borschensky and Reinacher, 2014; Olasz et al., 2017b).

FECVs have three open reading frames (ORFs) in region 3 (Fig. 1A). The $\sim 70$ amino acid $3 \mathrm{a}$ and $3 \mathrm{~b}$ proteins are conserved among subspecies 1 alphacoronaviruses. Because of the lack of predicted hydrophobic helices, both are thought to be localised in the cytoplasm, although their functions and their role in viral replication are unknown so far (Kipar and Meli, 2014).

The sequence of the $\sim 240$ residue ORF $3 \mathrm{c}$ suggests that it is a class III membrane protein (Kipar and Meli, 2014). It seems that intact, deletion-free 3c is needed for FECV to replicate in the gut (Chang et al., 2010; Bálint et al., 2014; Porter et al., 2014). Among the ORFs of region 3 ORF $3 \mathrm{c}$ is the best studied, yet experimental data regarding its localisation and function in the cells are scarce. It was found to be weakly expressed in Felis catus whole fetus-4 (Fcwf-4) cells and was suggested to localise in the endoplasmic reticulum (ER)/Golgi based on the similar distribution pattern found at the related transmissible gastroenteritis virus (TGEV) 3b protein (O'Connor and Brian, 1999; Hsieh et al., 2013).

To gain additional data about the function of the proteins of FECV region 3, we expressed them in different cell lines and investigated them in silico with different prediction programs.

\section{Materials and methods}

Total RNA was purified with QIAamp Viral RNA Mini Kit (Qiagen, Hilden, Germany) from an anal swab of an otherwise healthy FECV-positive cat showing signs of diarrhoea. Region 3 of FECV was amplified with the FIP_3abc Fw and FIP_3abc_R primers (Table 1) using the OneStep RT-PCR Kit (Qiagen, Hilden, Germany) and the amplicon was purified with Nucleospin gel purification kit (Macherey-Nagel, Düren, Germany) according to the manufacturer's recommendation.

The three ORFs of region 3 were amplified with six cloning primers (Ma3af-Ma3cr) (Table 1) by the Phusion Hot Start II DNA Polymerase (Thermo Fisher Scientific, Waltham, USA) using the sequenced region 3 amplicon as template. Fragments encoding ORF3a-c were digested with XhoI and PstI enzymes and cloned into pEGFP-N1(Clontech, Fermont, USA) and p-MeGFPN1 (Olasz et al., 2016) vectors in frame with the eGFP protein and into the pcDNAFLAG vector (Olasz et al., 2016). 
The viral amplicon was sequenced by BigDye Terminator sequencing kit (Thermo Fisher Scientific, Waltham, USA) using the amplifying primers and the MaCo3 primers (Table 1).

Table 1

Primers applied in the studies

\begin{tabular}{ll}
\hline Name & \multicolumn{1}{c}{ Sequence } \\
\hline FIP_3abc_Fw & 5'-AAT GGC CDT GGT ATG TGT-3' \\
FIP_3abc_R & 5'-CAT DCC ATK GTC RTC TAT-3' \\
Ma3af & 5'-GAC TGC TGG ATC CCT CGA GAC CAT GGA CAC TGT CAA GTC TAT TGG C-3' \\
Ma3ar & 5'-GAC TCT CGA GCT GCA GAA CAG TCG TAT GAT TAT GCG ACT C-3' \\
Ma3bf & 5'-GAC TGC TGG ATC CCT CGA GAC CAT GCC AAA CTT CAG CTG GAT ATT G-3' \\
Ma3br & 5'-GAC TCT CGA GCT GCA GTT TTC GCA CTG CGT TTA AAA TGG C-3' \\
Ma3cf & 5'-GAC TGC TGG ATC CCT CGA GAC CAT GAT TGG CGG ATT GTT CCT C-3' \\
Ma3cr & 5'-GAC TCT CGA GCT GCA GGG AAA CAT CAT AGA CAC GCG C-3' \\
MaCo3 & 5'-TAA AGC TAA GAC AGT TGT TG-3' \\
\hline
\end{tabular}

Plasmid constructs were transfected into CrFK, Fcwf-4 and MARC-145 cells with Turbofect (Thermo Scientific, Waltham, USA) on 6-well plates according to the manufacturer's recommendation. The tissue culture adapted FIPVDF2 strain (multiplicity of infection 3) was used for the infection of the transfected CrFK cells as it was described earlier (Bálint et al., 2012).

For immunofluorescence (IF) detection, the cells were fixed with $300 \mu 1$ $3 \%$ formaldehyde solution and were washed twice with $1 \mathrm{ml}$ washing buffer [phosphate-buffered saline (PBS) $0.1 \%$ Tween 20]. After washing, they were incubated in Hoechst $33342(1 \mu \mathrm{g} / \mu \mathrm{l})$ for $1 \mathrm{~h}$ and washed again twice with washing buffer. In case of the $3 \mathrm{c}$ and the FLAG-labelled $3 \mathrm{a}$ constructs, the cells were incubated in 1\% Triton-X for 30 min after fixation and (after washing) the ER and the Golgi intermediate compartment (ERGIC) of cells were stained using a 200fold diluted mouse anti-calreticulin IgG (Thermo Fisher Scientific, Waltham, USA), ERGIC-53(F-3) (Santa Cruz Biotechnology, Dallas, USA) and 100-fold diluted anti-ABCD IgG1 (Sigma-Aldrich, St. Louis, USA) for $1 \mathrm{~h}$, respectively. The cells were washed again and incubated in 500-fold diluted, CF586-labelled, anti-mouse IgG (Thermo Fisher Scientific, Waltham, USA) and in Hoechst $33342(1 \mu \mathrm{g} / \mu \mathrm{l})$ for $1 \mathrm{~h}$. To label intermediate-to-trans Golgi Lectin GS-II Alexa Fluor 647 conjugate (Thermo Fisher Scientific, Waltham, USA) was used in 300-fold dilution. Samples were examined under an Axio Observer D1 inverse fluorescence research microscope (Zeiss, Oberkochen, Germany).

For the immunoprecipitation of the eGFP fusion proteins, the Protein $G$ Immunoprecipitation Kit (Sigma-Aldrich, St. Louis, USA) was used. Transfected cells from a 6 -well plate were lysed in $600 \mu 1 \mathrm{xIP}$ buffer with $0.1 \%$ SDS. To bind the eGFP proteins, $10 \mu$ GFP Tag polyclonal antibody (Thermo Fisher Scientific, Waltham, USA) was added to the lysates. All other treatments to prepare the eGFP-tagged proteins for SDS-polyacrylamide gel electrophoresis (PAGE) 
were executed following the manufacturer's Basic Protocol recommendation. Immunoblot detection of the GFP fusion proteins on supported nitrocellulose membrane (Bio-Rad, Hercules, USA) after PAGE was carried out by using a monoclonal anti-eGFP antibody (Thermo Fisher Scientific, Waltham, USA) in a 500-fold dilution and horseradish-peroxidase-conjugated goat anti-mouse IgG (Southern Biotech, Birmingham, USA) in a 1000-fold dilution. The horseradish peroxidase activity was revealed with 3,3'-diaminobenzidine tetrahydrochloride (Sigma-Aldrich, St. Louis, USA) substrate according to the supplier's recommendations. The molecular weights of the expressed proteins were compared to the PageRuler Plus Protein Ladder (Thermo Fisher Scientific, Waltham, USA).

For protein function prediction, the NLS Mapper (Kosugi et al., 2009), NucPred (Brameier et al., 2007), NLStradamus (Nguyen Ba et al., 2009), Nine Amino Acids Transactivation Domain 9aaTAD Prediction Tool (Piskacek et al., 2007), MITOPROT (Claros and Vincens, 1996), PSFM-DBT (Zhang and Liu, 2017), DP-Bind (Kuznetsov et al., 2006; Hwang et al., 2007) and the DRNApred (Yan and Kurgan, 2017) web servers were used.

\section{Results}

Since there was no available monospecific or monoclonal antibody against the proteins of FECV region 3, we decided to study them as GFP- and 3xFLAGtagged fusion proteins. First the three ORFs were amplified by PCR and cloned into a pEGFP-N1 vector and transfected into Fcwf- 4 cells. After $36 \mathrm{~h}$ the cells were fixed and monitored under fluorescent microscope. Fluorescent signal could be detected inside the transfected cells indicating the translation of the GFP proteins. However, the localisation of the fusion proteins seemed to be very similar to that of the eGFP (nuclear and cytoplasmic) (Figs 2A-D), especially in the case of $3 \mathrm{a}$ and $3 \mathrm{c}$ (Figs $2 \mathrm{~A}$ and $\mathrm{C}$ ), though it was expected that at least the 3cGFP fusion protein would localise in some membranous compartment of the cytoplasm. To characterise the expressed GFP fusion proteins, transfected cells of all three constructs were lysed and the fusion proteins were immunoprecipitated by an anti-eGFP polyclonal antibody and their sizes were analysed on Western blot by an anti-eGFP monoclonal antibody. Surprisingly, bands of equal size to eGFP $(27 \mathrm{kDa})$ were detected in the lysates of all constructs (Fig. 2E). Moreover, they seemed to be the dominant bands except in the case of the 3a-eGFP construct, while no band with the expected size $(54 \mathrm{kDa})$ was detected in the case of the 3c-eGFP construct (Fig. 2E). These findings suggest that native eGFP is translated from the fusion constructs that interferes with the detection of the viral fusion proteins and that $3 \mathrm{c}$-eGFP is very weakly expressed in the transfected cells. To eliminate the interfering effect of the translated native eGFP, the three ORFs were cloned in the p-MeGFPN1 vector, which contains a mutation in the initia- 
tion codon of eGFP (Olasz et al., 2016), thereby eliminating native eGFP translation. In these fusion constructs translation can be initiated only from the ATGs of the cloned ORFs. This approach proved to be successful and clear, eGFP background-free signals could be detected in the transfected cells that allowed us to determine the localisation of the $3 \mathrm{a}, 3 \mathrm{~b}$ and $3 \mathrm{c}$ proteins (Figs 3, 4 and 5).

The 3 a protein does not contain any short basic stretches of amino acids or any recognisable classical nuclear localisation signals (NLS), and no prediction programs (NLS Mapper, NucPred, NLStradamus (Brameier et al., 2007; Kosugi et al., 2009; Nguyen Ba et al., 2009) recognise such motifs on them. Yet, the majority of the 3a-eGFP protein is localised in the nucleus, though it can also be detected in the cytoplasm (Fig. 3A). Since eGFP alone shows somewhat similar distribution in the CRFK cells (nuclear and cytoplasmic) (Fig. 3B), we also transfected a construct where the 3 a protein was fused to a $3 x$ FLAG-tag. Detection of the 3xFLAG-tag with anti-FLAG antibody verified the nuclear and the cytoplasmic localization of the 3a protein (Fig. 3C). The nuclear localisation of the protein can indicate a DNA- or RNA-binding function, so we screened the protein sequence of $3 \mathrm{a}$ for the presence of DNA- or RNA-binding domains by various prediction programs, and in fact some of the programs predicted potential DNA-binding amino acids in the 3 a protein (Fig. 1B). The high net negative charge (pI 4.41) and strong hydrophobic character of the protein suggested the presence of a transcription activator domain (TAD) on 3a. In fact, analysis of the sequence with the Nine Amino Acids Transactivation Domain 9aaTAD Prediction Tool (Piskacek et al., 2007) revealed not one but two regions between amino acids $12-20$ and $46-63$ that could serve as a potential TAD (Fig. 1B).

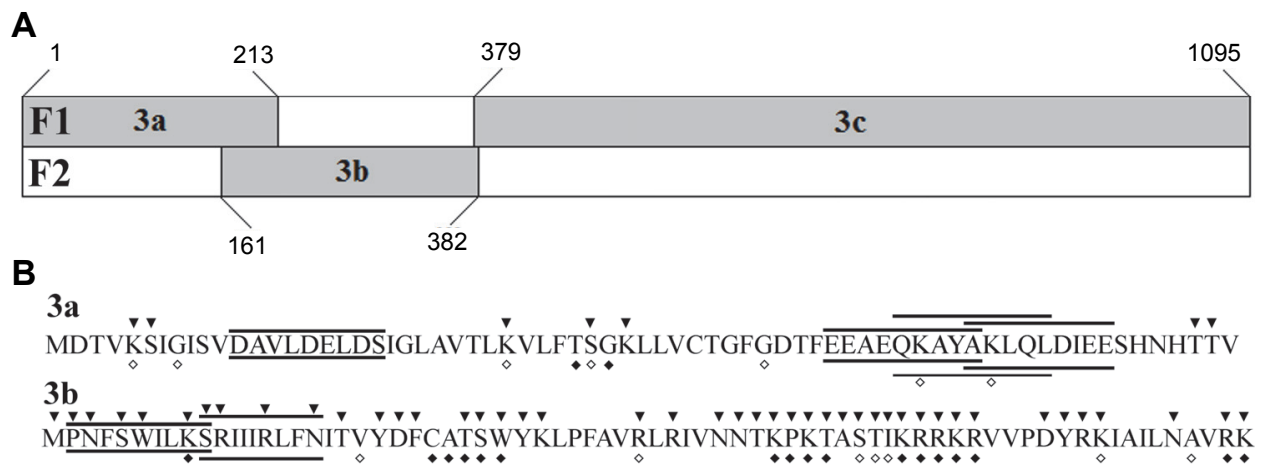

Fig. 1. Schematic representation of the region 3 of the Feline Coronavirus and the amino acid sequences of the $3 \mathrm{a}$ and $3 \mathrm{~b}$. (A) Reading frames are labelled by F1 and F2. The ORFs of region 3 are symbolised by shaded boxes, and the first and last nucleotides are numbered. (B) Predicted activator regions (by Nine Amino Acids Transactivation Domain 9aaTAD Prediction Tool) are underlined. The triangles and rhombuses indicate the amino acids potentially involved in DNA binding (predicted by the DNApred and DP-Bind programs, respectively) 

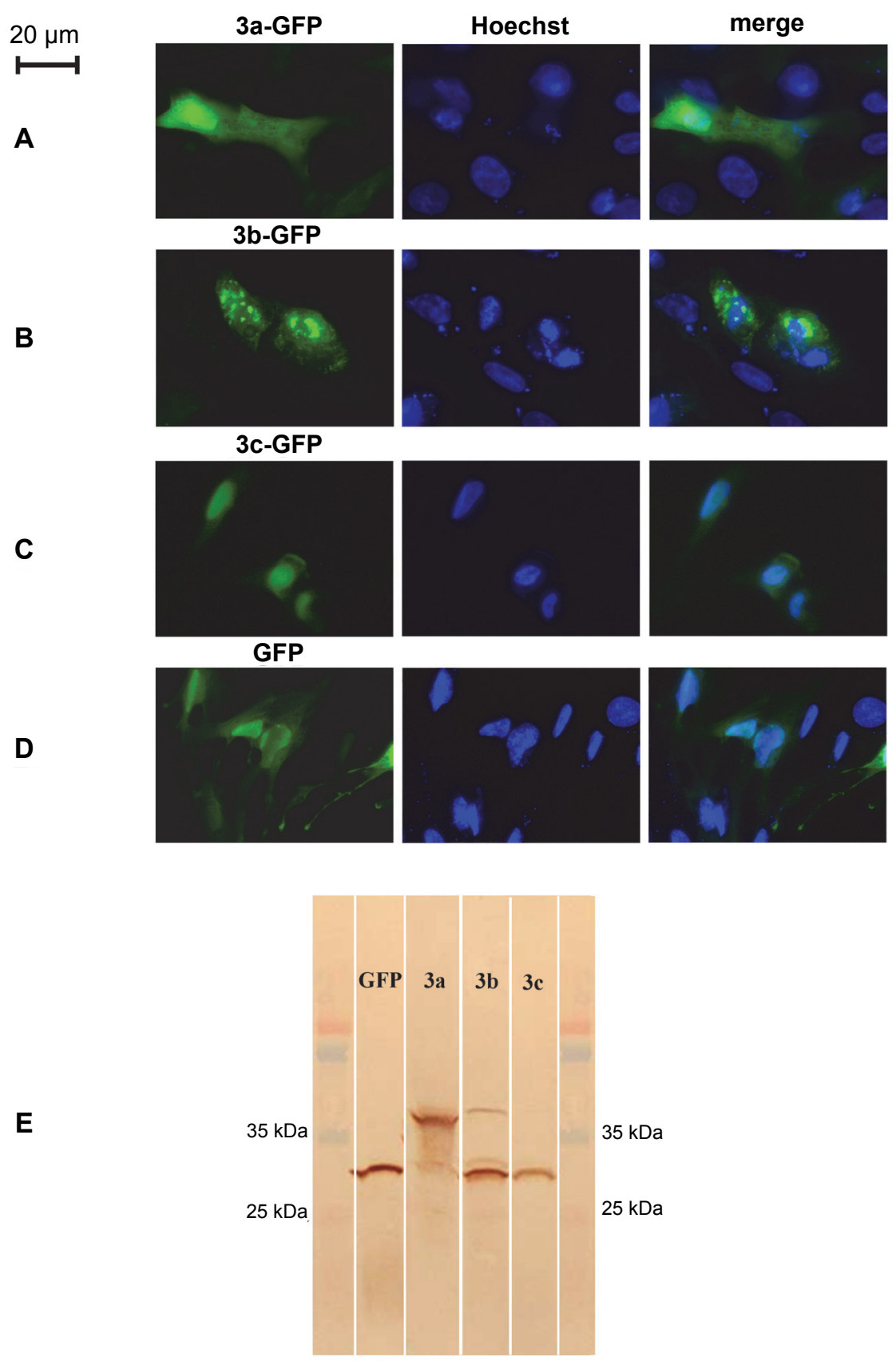

Fig. 2. Detection of eGFP (with start codon) fused region 3 proteins of FECV. (A) Immunofluorescent detection of 3a-eGFP, (B) 3b-eGFP, (C) 3c-eGFP and (D) eGFP proteins in Fwcf-4 cells at 36 $\mathrm{h}$ after transfection. Nuclei are labelled with Hoechst 33342. (E) Western blot detection of the expressed proteins 


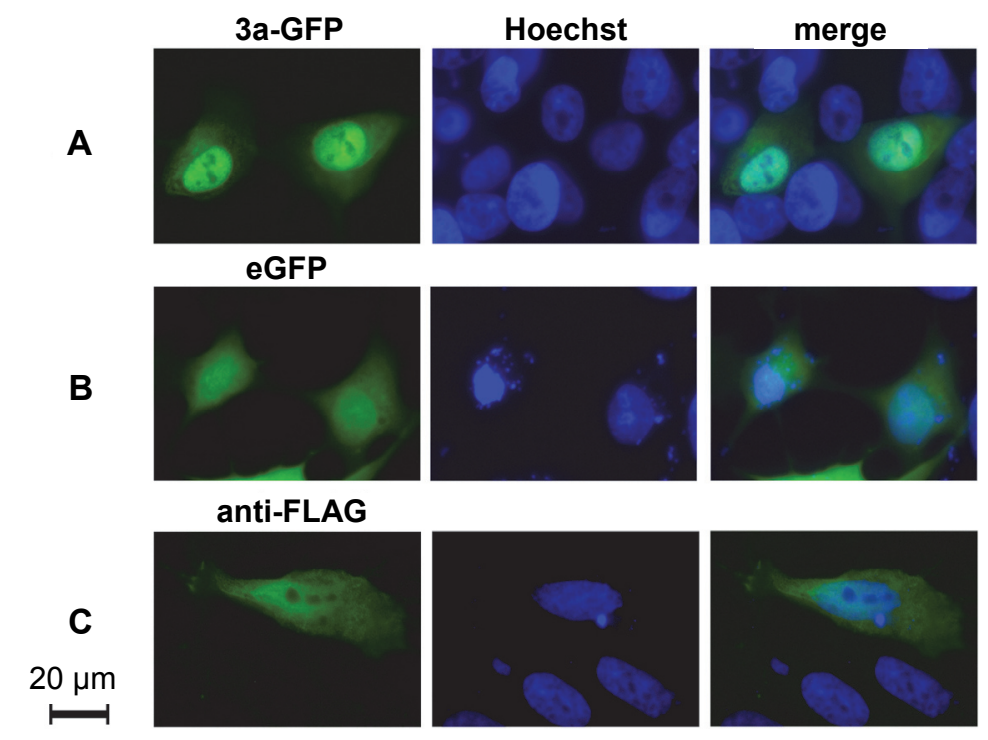

Fig. 3. Intracellular localisation of the 3a protein in CrFK cells after transfection. (A) 3a protein was expressed with fused eGFP (without start codon). Cells were fixed at $36 \mathrm{~h}$ post transfection. (B) eGFP-transfected CrFK cells. (C) Localisation of 3xFLAG tagged 3a, detected by anti-FLAG antibody. Cells were fixed at $20 \mathrm{~h}$ post transfection. Nuclei are labelled with Hoechst 33342

In the majority of the cells $3 \mathrm{~b}$-eGFP protein was localised as a granular/speckled pattern in the cytoplasm strongly resembling mitochondrial localisation (Fig. 4). When the 72-amino-acid-long sequence of $3 \mathrm{~b}$ was investigated by a computer program predicting mitochondrial transport (MITOPROT; Claros and Vincens, 1996), an almost perfect score (probability 0.9982) was gained, further supporting mitochondrial localisation. To gain fool-proof results, the 3b-eGFP and $3 b-3 x F L A G$ constructs were co-transfected with the pDsRed2-Mito plasmid expressing the mitochondrial human cytochrome-c oxidase protein fused to the dsRed fluorescent protein. In the co-transfected cells the green and the red fluorescent signal co-localised, verifying that the $3 \mathrm{~b}$ protein is indeed localised in the mitochondria (Fig. 4A-D and F). Besides the mitochondrial localisation, in around one-third of the transfected cells the $3 \mathrm{~b}$-eGFP protein was also clearly visible in the nucleolus (Fig. 4B-D). In a few transfected cells $(1 / 100)$ very tight perinuclear accumulation or possibly nuclear membrane localisation of the $3 \mathrm{~b}$ eGFP labelled protein was observed. However, perinuclear 3b-eGFP did not colocalise with either the pDsRed2-Mito or the cytochrome-c mitochondrial markers (Fig. 4C-D). In the same ways as for the 3a protein, potential DNA binding regions and two minimally overlapping TAD domains were predicted at the amino-terminal of the protein (Fig. 1B). 


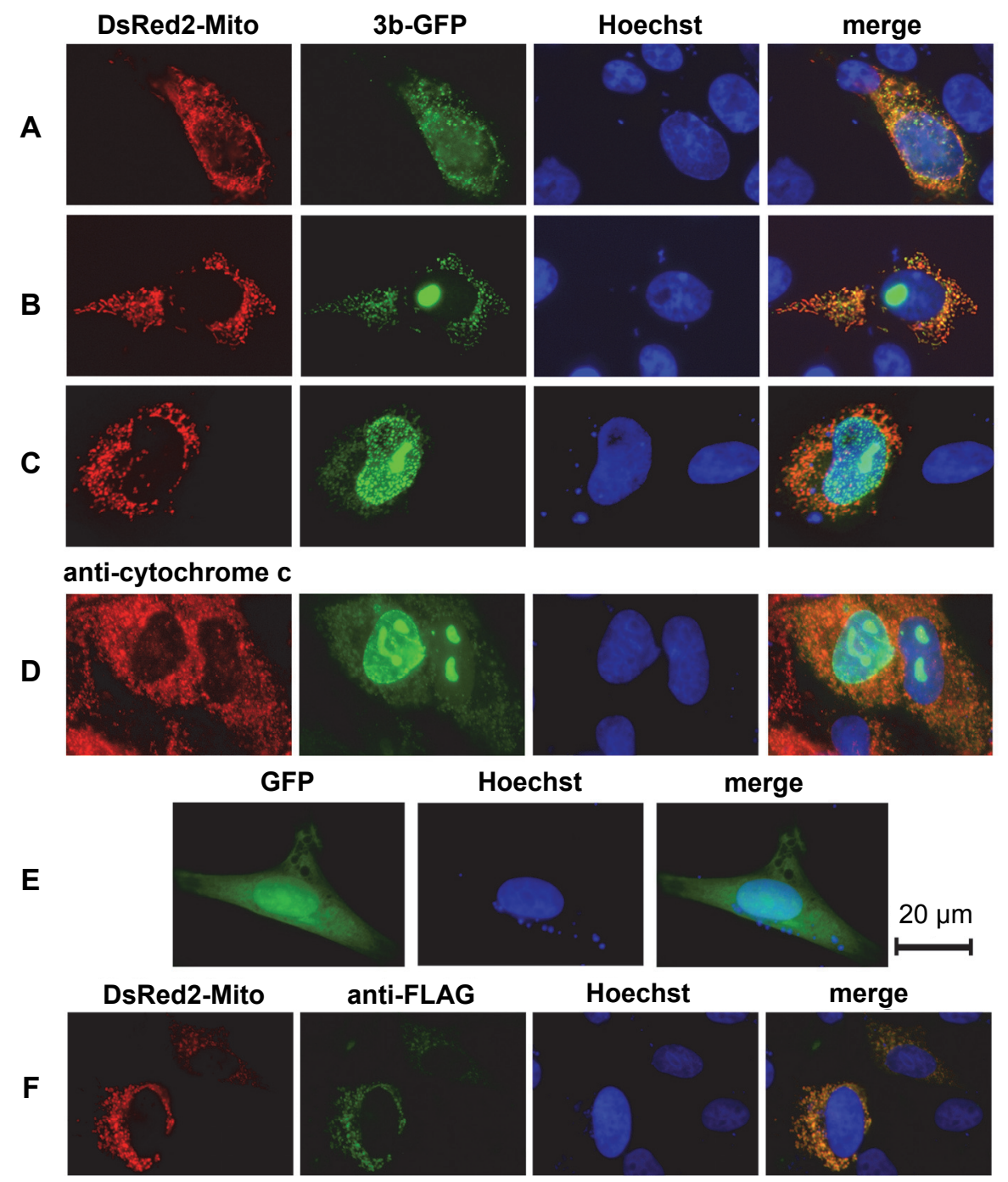

Fig. 4. Localisation of the $3 \mathrm{~b}$ protein in CRFK cells. (A-C) Localisations of the $3 \mathrm{~b}$-eGFP fusion protein (green channel) and DsRed2-Mito mitochondrial marker protein (red channel). (D) Colocalisation of the Cytochrom C and the 3b-eGFP fusion protein. (E) eGFP-transfected CrFK cells (F) Detection of the 3b-3xFLAG and the DsRed2-Mito mitochondrial marker protein. Nuclei are labelled with Hoechst 33342

The $3 \mathrm{c}$ protein showed very weak expression, and 3c-eGFP and 3c$3 x$ FLAG fusion proteins could be detected (Fig. 5) in only a very small fraction of the CrFK cells (less than one in the hundreds). In these cells the fluorescent signal is localised in small point-like structures diffused in the cytoplasm that seemed to aggregate in a larger structure near the nucleus. Interestingly, the GFP 
signal did not co-localise with the signal of the ER marker calreticulin (Fig. 5A), suggesting that $3 \mathrm{c}$ is not localised in the ER as it was suggested by Hsieh et al. (2013). Localisation of the $3 \mathrm{c}$ protein was also investigated in the intermediateto-trans Golgi, in the peroxisome and in the ERGIC by staining the transfected cat-originated cell lines with Lectin GS-II binding $\alpha$ - and $\beta$ - $N$-acetyl-Dglucosaminyl residues anti-ABCD3 (recognising peroxisome-resident $\mathrm{ABCD} 3$ protein) and anti-ERGIC antibodies. The $3 \mathrm{c}$ protein co-localised with neither the peroxisome nor the ERGIC markers (Figs 5C-E). Unfortunately, Lectin GS-II bound to neither Fcwf-4 nor CrFK cells (data not shown). To gain additional data about the localisation of $3 \mathrm{c}$ the $3 \mathrm{c}$-eGFP-expressing construct was transfected into MARC-145 cells (originating from African green monkey kidney) in which Golgi is readily stained by Lectin GS-II. Surprisingly, 3c was expressed much more strongly in MARC-145 cells than in the established cat cell lines (detected in one tenth of the cells), but it showed similar distribution and localisation. Anti-ERGIC labelling of the transfected MARC 145 cells confirmed that $3 \mathrm{c}$ is not located in the ERGIC (Fig. 5D), while Lectin GS-II labelling of the 3cexpressing cells revealed that the $3 \mathrm{c}$ protein does not localise in the Golgi either (Fig. 5B).

To investigate the effect of other viral proteins on the localisation and stability of the proteins of region $3, \mathrm{CrFK}$ cells were transfected with $3 \mathrm{a}-\mathrm{c}$ expressing plasmids and the cells were infected with FIPV-DF2 $6 \mathrm{~h}$ post transfection and fixed at $18 \mathrm{~h}$ post infection. At that time the effect of viral infection was clearly visible, manifesting itself in cytopathic effects, cell death and the formation of syncytia. Viral infection had no visible effect on the localisation of $3 \mathrm{a}$ and $3 \mathrm{c}$ either (Figs $6 \mathrm{~A}$ and $\mathrm{D}$ ), and $3 \mathrm{c}$ protein was expressed still very poorly in CrFK cells.

However, $3 \mathrm{~b}$ can be detected much more frequently $(\sim 50 \%)$ in the nucleolus of infected non-syncytial cells than in that of the non-infected cells (data not shown). In infected syncytial cells this difference cannot be observed. The perinuclear or nuclear membrane localisation of $3 b$ was also more frequent $(1 / 10)$ in syncytial cells (Figs 6B-C).

\section{Discussion}

Many comparative diagnostic and experimental observations implicate ORF3abc in the pathogenesis of FIP, yet very little is known about the exact functions of this genetic region (Haijema et al., 2007; Pedersen, 2009). In this study we determined the localisation of the proteins coded by the three ORFs of the region by tagging them with eGFP and 3xFLAG. Carboxy terminal tagging of the proteins was chosen because none of the utilised protein function and signal prediction programs predicted such functional units whose interaction with the carboxy terminal tag could potentially interfere with the localisation of proteins. The distribution of $3 \mathrm{a}-\mathrm{c}$ was similar in the cells with both tags, suggesting 


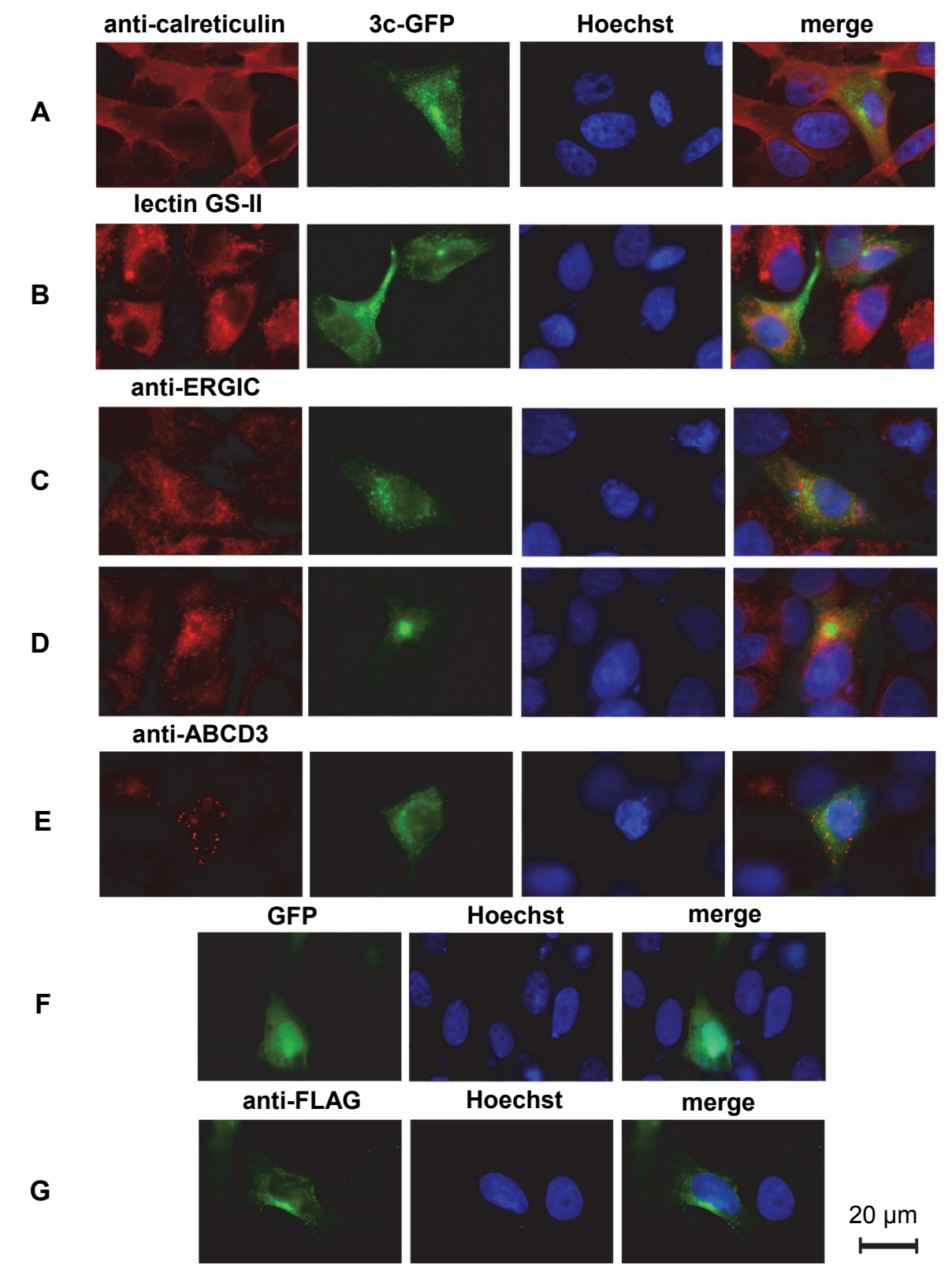

Fig. 5. Intracellular localisation of the $3 \mathrm{c}$ protein in MARC-145 and CrFK cells at $36 \mathrm{~h}$ post transfection. (A) The transfected CrFK cells were stained with anti-calreticulin antibodies (ER marker). (B) The transfected MARC-145 cells were labelled by Lectin GS-II conjugated with Alexa Fluor 647 (trans-Golgi marker). ERGIC-53(F-3) antibody was used for the detection of ERGIC in 3c-eGFP expressing (C) CrFK and (D) MARC-145 cells. (E) Anti-ABCD3-labelled peroxisome and 3c-eGFP in CrFK cells. (F) eGFP-transfected CrFK cells. (G) Localisation of 3c-3xFLAG in CRFK cells. Nuclei are labelled with Hoechst 33342 

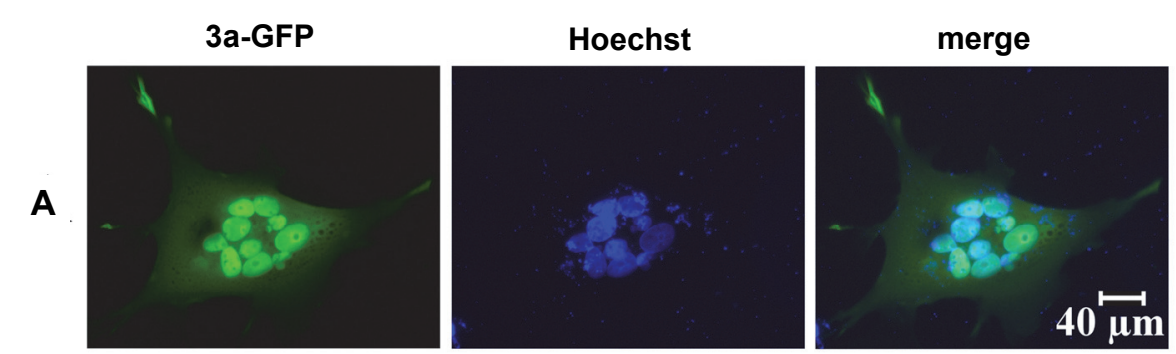

3b-GFP

B
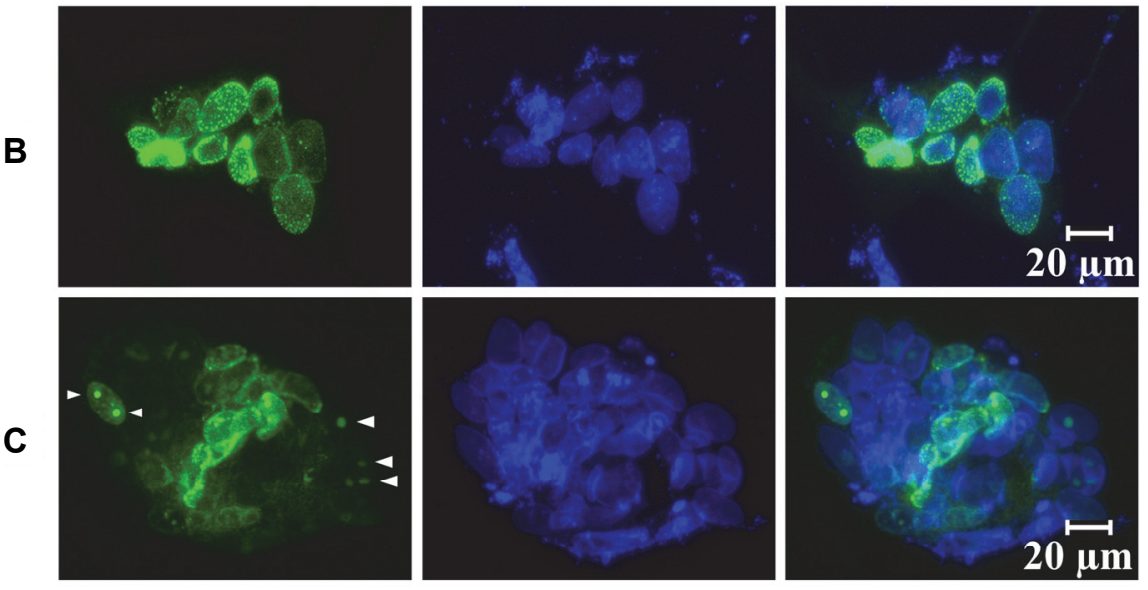

3c-GFP
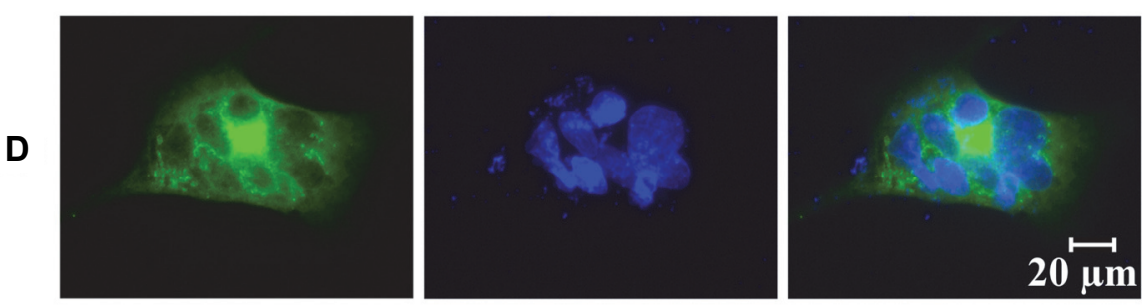

GFP
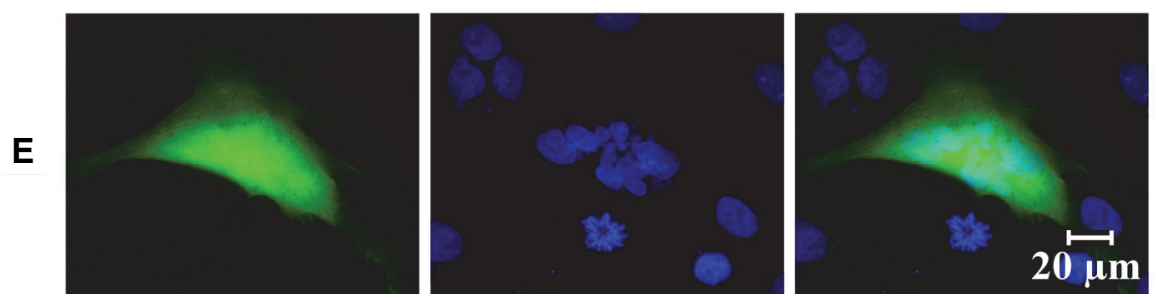

Fig. 6. Intracellular localisation of the eGFP fused region 3 proteins of FECV in FIPV-infected CrFK cells at $24 \mathrm{~h}$ post transfection, $18 \mathrm{~h}$ post infection. (A) $3 \mathrm{a}-\mathrm{eGFP}$ expressing FIPV infected cells. (B and C) 3b-eGFP-expressing FIPV-infected cells. The arrows show the accumulated $3 \mathrm{~b}$ protein in the nucleolus. (D) Localisation of the 3c-eGFP in infected cells. (E) eGFP-expressing FIPV infected cells. Nuclei are labelled with Hoechst 33342 
that the fusion partners do not influence artificially the localisations of the proteins. Despite these findings it cannot be excluded that the investigation of the native proteins with specific antibodies may reveal additional and more defined information about the function and localisation of these proteins.

Viral RNAs are widely known for non-canonical translation initiation (it is not only the first ATG that serves as an initiation codon on the mRNA), and several mechanisms (e.g. leaky scanning, shunting, IRES, etc.) were identified in viral protein synthesis (O'Connor and Brian, 2000; Firth and Brierley, 2012). Transient expression of the eGFP fusion constructs (made in pEGFP-N1 plasmids with functional eGFP initiation codon) in all cases resulted in high levels of native eGFP, that were detected in the cytoplasm and the nucleus of the transfected cells by immunofluorescence microscope and by Western blot (Fig. 2). This result suggested that in all investigated FECV ORFs alternative initiation occurs and at least the first ATG codon downstream of these ORFs is able to initiate translation. Expressing the ORFs as eGFP fusion protein without functional eGFP initiation codon (in p-MeGFPN1 plasmid, a mutated derivative of pEGFPN1) verified this suspicion, since the native eGFP background disappeared from the cytoplasm and from the nucleus of cells transfected with $3 b$ - and 3c-eGFP constructs (Figs 4 and 5). Furthermore, the finding that an internal entry mechanism is used for the expression of ORF $3 \mathrm{~b}$ in the closely related TGEV (O'Connor and Brian, 2000) suggests that non-canonical translation initiation is generally used to translate the consecutive ORFs of the region 3 in alphacoronavirus 1 species (FECV, CCoV, TGEV, etc.). The determination of the exact noncanonical mechanisms contributing to the translation of the region requires further investigations.

ORFs $3 \mathrm{a}$ and $3 \mathrm{~b}$ are exclusive features of the subspecies of the alphacoronavirus 1 species. Proteins translated from the two ORFs were suggested to locate in the cytoplasm based on their predicted physicochemical characteristics (Kipar and Meli, 2014). However, our experimental evidence contradicted this presumption.

Protein 3a is localised in the nucleus and in the cytoplasm (Figs $3 \mathrm{~A}$ and $\mathrm{C}$ ). Since it does not contain NLS, it is most probably transported to the nucleus by diffusion or complex formation (piggyback transport) with cellular proteins containing NLS. The localisation of the protein in multiple cellular compartments and the presence of predicted DNA binding and activator domains indicate pleiotropic functions of $3 \mathrm{a}$ that most probably involve the reprogramming of the transcriptional regulation of the infected cells. Viral proteins with similar multiple (cytosolic and nuclear) localisations were identified in several different viruses (e. g. 7ap of PRRSV, X protein of hepatitis B virus, $\mu 2$ of reovirus T1L (Mbisa et al., 2000; Henkler et al., 2001; Ma et al., 2011; Olasz et al., 2016, $2017 a$; Rivera-Serrano et al., 2017) with proven nucleic acid binding and/or transcription regulating capabilities. 
Protein $3 \mathrm{~b}$ was found to localise in the mitochondrion and the nucleolus (Fig. 4). With this feature, $3 \mathrm{~b}$ is not unique among coronaviral accessory proteins. The non-homologous 154-aa-long $3 \mathrm{~b}$ protein of the SARS-CoV, that is translated from an entirely differently organised region 3 , was shown to localise in the mitochondria and in the nucleolus, and to inhibit type I interferon (IFN I) production (Yuan et al., 2006). Besides its involvement in innate immunity modulation, the mitochondrial localisation of $3 \mathrm{~b}$ may suggest an anti- or proapoptotic function of $3 \mathrm{~b}$. Since the mitochondrial pathway represents the main intrinsic signalling cascade for programmed cell death (Benedict et al., 2002), several viruses (e.g. $\alpha$-herpesviruses, adenoviruses, hepatitis $\mathrm{C}$ virus, influenza A virus) manipulate this pathway for their own advantage (White, 1998; Chung et al., 2003; You et al., 2017; Yeganeh et al., 2018). The nucleolar localisation and presence of potential activator and DNA binding domains suggest a role of $3 \mathrm{~b}$ in the regulation of translation through the influence of ribosome biogenesis in the infected cells. In some of the non-infected and in the FIPV-infected cells perinuclear or nuclear membrane accumulation of the $3 \mathrm{~b}$ was observed (Figs 4B-D and $6 \mathrm{~B}-\mathrm{C})$. The distribution of the $3 \mathrm{~b}$ around the nucleus suggests an interaction of the protein with the nuclear pores or with the nuclear lamina. There are numerous reports about the interaction of the proteins of DNA viruses (e.g. herpesviruses and polyomaviruses) with the nuclear lamina (Cibulka et al., 2012) but (to our knowledge) such interaction has not yet been reported about the proteins of cytoplasmic (cp) RNA viruses. However, interaction was found among the proteins of several cpRNA viruses (Venezuelan equine encephalitis virus, picornaviruses) and the nuclear pore complex to regulate nucleocytoplasmic trafficking (Younessi et al., 2012; Le Sage and Mouland, 2013; Yarbrough et al., 2014). These findings make it more likely that the $3 \mathrm{~b}$ protein interacts with components of the nuclear pore. The fact that $3 \mathrm{~b}$ alone shows perinuclear accumulation (Figs $4 \mathrm{C}-\mathrm{D}$ ) only in a few of the non-infected cells makes it highly probable that other viral protein(s) is/are also involved in the accumulation process during viral infection. The different ratios of $3 \mathrm{~b}$ in the cellular compartments (mitochondrial, perinuclear and nucleolar) in the different stages of the cells (infected, noninfected and syncytial) could be the consequence of changing interactions of $3 \mathrm{~b}$ with cellular and viral proteins during the successive steps of infection. However, further investigations are needed to clarify these presumptions.

The $3 \mathrm{c}$ protein of FECV is predicted to be a membrane protein and it is an orthologue ( $81 \%$ amino acid identity) of the $3 \mathrm{~b}$ of TGEV. Both $3 \mathrm{c}$ and $3 \mathrm{~b}$ of TGEV were shown to be very weakly expressed or instable in tissue culture cells and cannot be detected by Western blot (O'Connor and Brian, 2000; Hsieh et al., 2013). Based on their fuzzy perinuclear localisation by IF, they were suggested to be localised in the ER-Golgi network. Our 3c-eGFP Western blot and IF experiments confirmed weak expression/instability of 3c in Fcwc-4 and CrFK cells. The exact compartmental localisation of $3 \mathrm{c}$ remained an enigma, it does not seem 
to be localised in the ER-Golgi network, ERGIC or peroxisomes (Fig. 5). It was also shown by others that it is not involved in autophagy (Hsieh et al., 2013). However, its localisation in the cytoplasmic compartments and aggregation in perinuclear region might indicate shuttling among or fusion of subcellular compartments. Interestingly, its expression in cell lines of non-host (non-human primate) origin largely increased the detectability of the 3c-eGFP protein by IF, raising the possibility that the quantity of the native $3 \mathrm{c}$ protein during FECV infection might also be different in host target cells (macrophages and enterocytes), and this difference may contribute to the deletion of the $3 \mathrm{c}$ region of the macrophage-specific FIPV (e.g. 3c might be overexpressed in enterocytes for an unknown biological function but underexpressed in macrophages and the deletion of its gene in FIPV does not cause further loss of biological function, because the coded functional protein is not there). Unfortunately, until further evidence emerges, these remarks remain only speculations, but our findings clearly indicate the necessity of in situ immunological investigations of $3 \mathrm{c}$ in native enterocytes and macrophages of FECV-infected cats.

\section{Acknowledgements}

The study was supported by the Hungarian Scientific Research Fund (K108607) and the National Research, Development and Innovation Office (K119381).

\section{References}

Bálint, Á., Farsang, A., Zádori, Z., Hornyák, Á., Dencso, L., Almazán, F., Enjuanes, L. and Belák, S. (2012): Molecular characterization of feline infectious peritonitis virus strain DF-2 and studies of the role of ORF3abc in viral cell tropism. J. Virol. 86, 6258-6267.

Bálint, Á., Farsang, A., Zádori, Z. and Belák, S. (2014): Comparative in vivo analysis of recombinant type II feline coronaviruses with truncated and completed ORF3 region. PLoS One 9, e88758.

Benedict, C. A., Norris, P. S. and Ware, C. F. (2002): To kill or be killed: viral evasion of apoptosis. Nat. Immunol. 3, 1013-1018.

Borschensky, C. M. and Reinacher, M. (2014): Mutations in the 3c and 7b genes of feline coronavirus in spontaneously affected FIP cats. Res. Vet. Sci. 97, 333-340.

Brameier, M., Krings, A. and MacCallum, R. M. (2007): NucPred - Predicting Nuclear Localization of Proteins. Bioinformatics 23, 1159-1160.

Chang, H. W., de Groot, R. J., Egberink, H. F. and Rottier, P. J. (2010): Feline infectious peritonitis: insights into feline coronavirus pathobiogenesis and epidemiology based on genetic analysis of the viral 3c gene. J. Gen. Virol. 91, 415-420.

Chung, Y. L., Sheu, M. L. and Yen, S. H. (2003): Hepatitis C virus NS5A as a potential viral Bcl-2 homologue interacts with Bax and inhibits apoptosis in hepatocellular carcinoma. Int. J. Cancer 107, 65-73.

Cibulka, J., Fraiberk, M. and Forstova, J. (2012): Nuclear actins and lamins in viral infections. Viruses $4,325-347$. 
Claros, M. G. and Vincens, P. (1996): Computational method to predict mitochondrially imported proteins and their targeting sequences. Eur. J. Biochem. 241, 779-786.

Firth, A. E. and Brierley, I. (2012): Non-canonical translation in RNA viruses. J. Gen. Virol. 93, $1385-1409$.

Haijema, B. J., Rottier, P. J. and de Groot, J. R. (2007): Feline coronaviruses: a tale of two-faced types. In: Thiel, V. (ed.) Coronaviruses: Molecular and Cellular Biology. Caister Academic Press, Norfolk, United Kingdom. pp. 183-203.

Henkler, F., Hoare, J., Waseem, N., Goldin, R. D., McGarvey, M. J., Koshy, R. and King, I. A. (2001): Intracellular localization of the hepatitis B virus HBx protein. J. Gen. Virol. 82, 871-882.

Herrewegh, A. A., Smeenk, I., Horzinek, M. C., Rottier, P. J. and de Groot, R. J. (1998): Feline coronavirus type II strains 79-1683 and 79-1146 originate from a double recombination between feline coronavirus type I and canine coronavirus. J. Virol. 72, 4508-4514.

Hsieh, L. E., Huang, W. P., Tang, D. J., Wang, Y. T., Chen, C. T. and Chueh, L. L. (2013): 3C protein of feline coronavirus inhibits viral replication independently of the autophagy pathway. Res. Vet. Sci. 95, 1241-1247.

Hwang, S., Gou, Z. and Kuznetsov, I. B. (2007): DP-Bind: a web server for sequence-based prediction of DNA-binding residues in DNA-binding proteins. Bioinformatics 23, 634-636.

Kipar, A. and Meli, M. L. (2014): Feline infectious peritonitis: still an enigma? Vet. Pathol. 51, 505-526.

Kosugi, S., Hasebe, M., Tomita, M. and Yanagawa, H. (2009): Systematic identification of yeast cell cycle-dependent nucleocytoplasmic shuttling proteins by prediction of composite motifs. Proc. Natl Acad. Sci. USA 106, 10171-10176.

Kuznetsov, I. B., Gou, Z., Li, R. and Hwang, S. (2006): Using evolutionary and structural information to predict DNA-binding sites on DNA-binding proteins. Proteins: Struct. Funct. Bioinf. 64, 19-27.

Le Sage, V. and Mouland, A. J. (2013): Viral subversion of the nuclear pore complex. Viruses 5, 2019-2042.

Ma, J., Sun, T., Park, S., Shen, G. and Liu, J. (2011): The role of hepatitis B virus X protein is related to its differential intracellular localization. Acta Biochim. Biophys. Sin. (Shanghai) 43, 583-588.

Mbisa, J. L., Becker, M. M., Zou, S., Dermody, T. S. and Brown, E. G. (2000): Reovirus mu2 protein determines strain-specific differences in the rate of viral inclusion formation in L929 cells. Virology 272, 16-26.

Nguyen Ba, A. N., Pogoutse, A., Provart, N. and Moses, A. M. (2009): NLStradamus: a simple Hidden Markov Model for nuclear localization signal prediction. BMC Bioinformatics 29, 202.

O'Connor, J. B. and Brian, D. A. (1999): The major product of porcine transmissible gastroenteritis coronavirus gene $3 \mathrm{~b}$ is an integral membrane glycoprotein of $31 \mathrm{kDa}$. Virology $\mathbf{2 5 6}$, $152-161$.

O'Connor, J. B. and Brian, D. A. (2000): Downstream ribosomal entry for translation of coronavirus TGEV gene 3b. Virology 269, 172-182.

Olasz, F., Dénes, B., Bálint, Á., Magyar, T., Belák, S. and Zádori, Z. (2016): Immunological and biochemical characterisation of 7ap, a short protein translated from an alternative frame of ORF7 of PRRSV. Acta Vet. Hung. 64, 273-287.

Olasz, F., Dénes, B., Bálint, Á., Magyar, T., Belák, S. and Zádori, Z. (2017a): Characterisation of the nucleic acid binding features of the PRRSV 7ap and its ability to induce antinuclear antibodies. Acta Vet. Hung. 65, 124-134.

Olasz, F., Kádár-Hürkecz, E., Bálint, Á., Lakatos, B. and Zádori, Z. (2017b): The biology of the feline infectious peritonitis virus (FIPV). Magy. Allatorvosok 139, 361-376.

Pedersen, N. C. (2009): A review of feline infectious peritonitis virus infection: 1963-2008. J. Feline Med. Surg. 11, 225-258. 
Piskacek, S., Gregor, M., Nemethova, M., Grabner, M., Kovarik, P. and Piskacek, M. (2007): Nine-amino-acid transactivation domain: establishment and prediction utilities. Genomics 89, 756-768.

Porter, E., Tasker, S., Day, M. J., Harley, R., Kipar, A., Siddell, S. G. and Helps, C. R. (2014): Amino acid changes in the spike protein of feline coronavirus correlate with systemic spread of virus from the intestine and not with feline infectious peritonitis. Vet. Res. 45, 49.

Rivera-Serrano, E. E., Fritch, E. J., Scholl, E. H. and Sherry, B. (2017): A cytoplasmic RNA virus alters the function of the cell splicing protein SRSF2. J. Virol. 91, e2488-16.

White, E. (1998): Regulation of apoptosis by adenovirus E1A and E1B oncogenes. Semin. Virol. 8, $505-513$.

Yan, J. and Kurgan, L. A. (2017): DRNApred, fast sequence-based method that accurately predicts and discriminates DNA- and RNA-binding residues. Nucl. Acids Res. 45, e84.

Yarbrough, M. L., Mata, M. A., Sakthivel, R. and Fontoura, B. M. (2014): Viral subversion of nucleocytoplasmic trafficking. Traffic 15, 127-140.

Yeganeh, B., Ghavami, S., Rahim, M. N., Klonisch, T., Halayko, A. J. and Coombs, K. M. (2018): Autophagy activation is required for influenza A virus-induced apoptosis and replication. Biochim. Biophys. Acta 1865, 364-378.

You, Y., Cheng, A. C., Wang, M. S., Jia, R. Y., Sun, K. F., Yang, Q., Wu, Y., Zhu, D., Chen, S., Liu, M. F., Zhao, X. X. and Chen, X. Y. (2017): The suppression of apoptosis by $\alpha$ herpesvirus. Cell Death Dis. 8, e2749.

Younessi, P., Jans, O. A. and Ghildyal, R. (2012): Modulation of host cell nucleocytoplasmic trafficking during picornavirus infection. Infect. Disorders Drug Targets 12, 59-67.

Yuan, X., Shan, Y., Yao, Z., Li, J., Zhao, Z., Chen, J. and Cong, Y. (2006): Mitochondrial location of severe acute respiratory syndrome coronavirus $3 \mathrm{~b}$ protein. Mol. Cells 21, 186-191.

Zhang, J. and Liu, B. (2017): PSFM-DBT: Identifying DNA-binding proteins by combing position specific frequency matrix and distance-bigram transformation. Int. J. Mol. Sci. 18, E1856. 